\title{
Mapping knowledge structure of artificial intelligence research in Bangladesh based on co-word analysis
}

\author{
Rajesh Kumar Das \\ Noakhali Science and Technology University, Noakhali, Bangladesh \\ Mohammad Sharif Ul Islam \\ University of Dhaka, Dhaka, Bangladesh
}

Article type

Research Paper

Received

Revised

15 November 2021

Accepted

10 December 2021
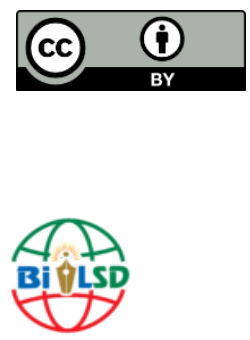

International Journal of Information and Knowledge Studies, 1(1), pp. 61-76

ISSN 2789-5920

https://doi.org/10.54857/ijiks.v1i1.6

Published by Bangladesh Institute for Information Literacy and Sustainable Development
10 June 2021

\begin{abstract}
Purpose: This article aims to map the knowledge structure of artificial intelligence (AI) in Bangladesh through detecting the interdisciplinarity and topic hotspots in the light of co-word analysis. Methodology: This study adopted bibliometric analysis of publications collected from the Web of Science (WoS) database. The WoS database was searched and 1557 publications were found. 1359 papers were selected for final analysis after eliminating duplicates. Co-occurrence words matrix, keyword clusters, hot topics were mapped using co-word analysis. The results were mapped, clustered and presented by VOSviewer.

Results: The result showed a rapidly increasing publication trajectory with 12 sub-domain cluster under the AI knowledge domain in Bangladesh. It also identified that AI, machine learning, classification, neural network, deep learning, artificial neural network, convolutional neural network, support vector machine and data mining are hot topics during the period of studied time. However, the findings also suggest that many research areas in the research domain of AI of Bangladesh is still nascent.

Limitation: VOSviewer often avoid having overlapping terms when multiple terms are positioned very close to each other. So, the overlapping terms remain invisible sometimes.

Practical implications: This study may have potential usefulness in uncovering the AI research fields' intellectual structure within a discipline and also to anticipate future innovation pathways of AI field in Bangladesh.

Originality: Bibliometric methods to explore the research trend and growth of AI research field as a 'knowledge base' in Bangladesh is one of the first attempts.
\end{abstract}

Keywords: Artificial intelligence, AI, Bibliometrics, Co-word analysis, Visualization, Bangladesh. 


\section{Introduction and background of the study}

The emergence of big data, the rise of the information citizenry and Internet of Things (IoT), the mass proliferation of the Internet along with the huge investment by the leading technology companies, such as IBM, Google, Amazon, and Facebook, have accelerated the growth of 'Artificial Intelligence (AI)' in both physical and virtual spaces (Li et al., 2017). John McCarthy defines AI as "the science and engineering of making intelligent machines" (McCarthy et al., 2007). The central goals of AI are to reason, discover, generalize, manipulate objects and natural language processing etc. (Copeland, 1993; Luger, 2005; Russell \& Norvig, 2002; Poole et al., 1998; Nilsson, 1998). Since its birth as a discipline in 1956, artificial intelligence (AI) has been one of the most dominant areas of research in which the scientific world is immersed into (Lungarella et al., 2007). AI has been a matter of great interest for years in varied disciplines like computer science, psychology, mathematics, information science, linguistics, and other specialized domains. AI is being successfully used to address multifaceted societal aspects through innovation and discovery in every domain. It expands into a broader spectrum of principles, tools, and techniques to help people to get the benefits from it even in their daily lives. Recently, AI and related technologies have influenced a lot of scholars and practitioners from science and industry to contribute to the field (Fosso et al., 2020). This wide-scale acceptability and usage have influenced academic and researcher of other domains and sub-domains to get involved from a multidisciplinary perspective (Chen, 2006).

By incorporating bibliometrics in general, and proper mapping techniques, analytic tools along with visualizations to be specific, could help to identify and represent the knowledge structure of a research field (Chen, 2013; Chen et al., 2015) of a domain. For clarification, bibliometrics, one of the major scientific mapping methods, is now widely being used to get the particular topics and subject categories excerpted from title, keyword and abstract (Van Eck \& Waltman, 2010). Many researchers use bibliometrics to map knowledge structure from various angles, such as co-word analysis (Callon et al., 1986; Rip \& Courtial, 1984), co-author analysis (Peters \& Van Raan, 1991), citation analysis (Garfield, 1972), social network analysis (Hummon \& Dereian, 1989) and so on. Among them, co-word analysis has gained interest among the researchers over the last couple of years, and has been studied in different fields, e. g., biotechnology (Chen, 2006; Rip \& Courtial, 1984; Cambrosio et al., 1993), polymer chemistry (Kevork \& Vrechopoulos, 2009) education (Ritzhaupt et al., 2010), Library and/ or Information Science (Liu et al., 2012; Coulter et al., 1998; Ding et al., 2001; Dong, 2009; Su, 2009; Wang et al., 2011) and so on.

This particular study has employed co-word analysis techniques to identify and visualize the major works of artificial intelligence submerged into various disciplines with a particular focus on Bangladesh. In short, 'Key words' are the lifeline of the co-word analysis procedure. Keywords are generally nouns or phrases that play a vital role as representatives of scholarly research publications. (Deka \& Sarmah, 2020; Xiang et al., 2017). It helps to describe the content of a paper succinctly, and thus useful to understand the similarity 
between two documents through the analysis of relatedness between keywords extracted from the title or abstract or full-text (Rip \& Courtial, 1984; Rokaya et al., 2008; Hu \& Zhang, 2015). Such type of cognitive connections among a set of documents constructs the basis of co-word analysis (Eom, 2008). Co-word analysis is a well-established technique employed for analyzing the intellectual structure of different scientific domains and research fields (Ronda-Pupo \& Guerras-Martin, 2012; Khasseh et al., 2017). Through co-word analysis, one could determine research theme, associations with them, semantic structure and direction of a field over time (Jacobs et al., 2006; Qiu et al., 2017; Hu et al., 2013). There are many techniques for co-word analysis. In this study, we conduct a co-word analysis using the method of keyword clustering and social network analysis (SNA).

\section{Rationale and objective of the study}

The widespread application and acceptability of AI has also gained a considerable amount of interest among the scientific communities in Bangladesh like other countries. However, the understanding of the multidisciplinary AI-based research, and its topic distribution from the country's perspective, have been lacking from the scientific publications. Thus, this paper aims to map the knowledge structure of artificial intelligence in Bangladesh during 1993-2020 through detecting the inter-disciplinarily and topic hotspots in the light of coword analysis. The research questions include-

- RQ1: What is the trend of artificial intelligence (AI) research in Bangladesh in terms of publication and citation?

- RQ2: How the publications are scattered into different discipline or research domains?

- RQ3: Which are the hot topics on AI during 1993-2020?

\section{Materials and methods}

\subsection{Data collection}

This study focused on the analysis of publications from the 'Web of Science' (WoS) database. For retrieving the papers, the following search strategy was developed based on the established keywords extracted from previous works:

$\mathrm{TS}=$ ("artificial intelligence" OR "machine intelligence" OR "machine learning" OR "deep learning" OR "natural language process" OR "neural network" OR "natural language process*” OR “expert system*" OR "intelligent system”) AND CU= Bangladesh

On 28 March 2021, the WoS database was searched using the above-mentioned search strings, and 1557 publications were found. After the elimination of the duplicates and those having no keywords, 1359 publications were selected for the final analysis. From exported records publication year, publication type, citation and subject categories were used to carry 
IJIKS, Vol. 1 No. 1 (2021)

out the bibliometric analysis (co-word analysis) using the MS Excel. All author keywords (DE) extracted from WOS has been analysed by incorporating VOSviewer 1.6.16.

\subsection{Data analysis}

For the co-word analysis co-occurrence words matrix, keyword clusters, hot topics were mapped, clustered and presented by VOSviewer version 1.6.16. VOSviewer, a web-based program (available from http://vosviewer.com) is being popularly used for conducting bibliometric analysis. It also has zooming and scrolling functionalities that help to be more visible and label overlap of large datasets. In this study, cluster analysis was grouped based on document-weights and both cluster view and density view have been used for visualization.

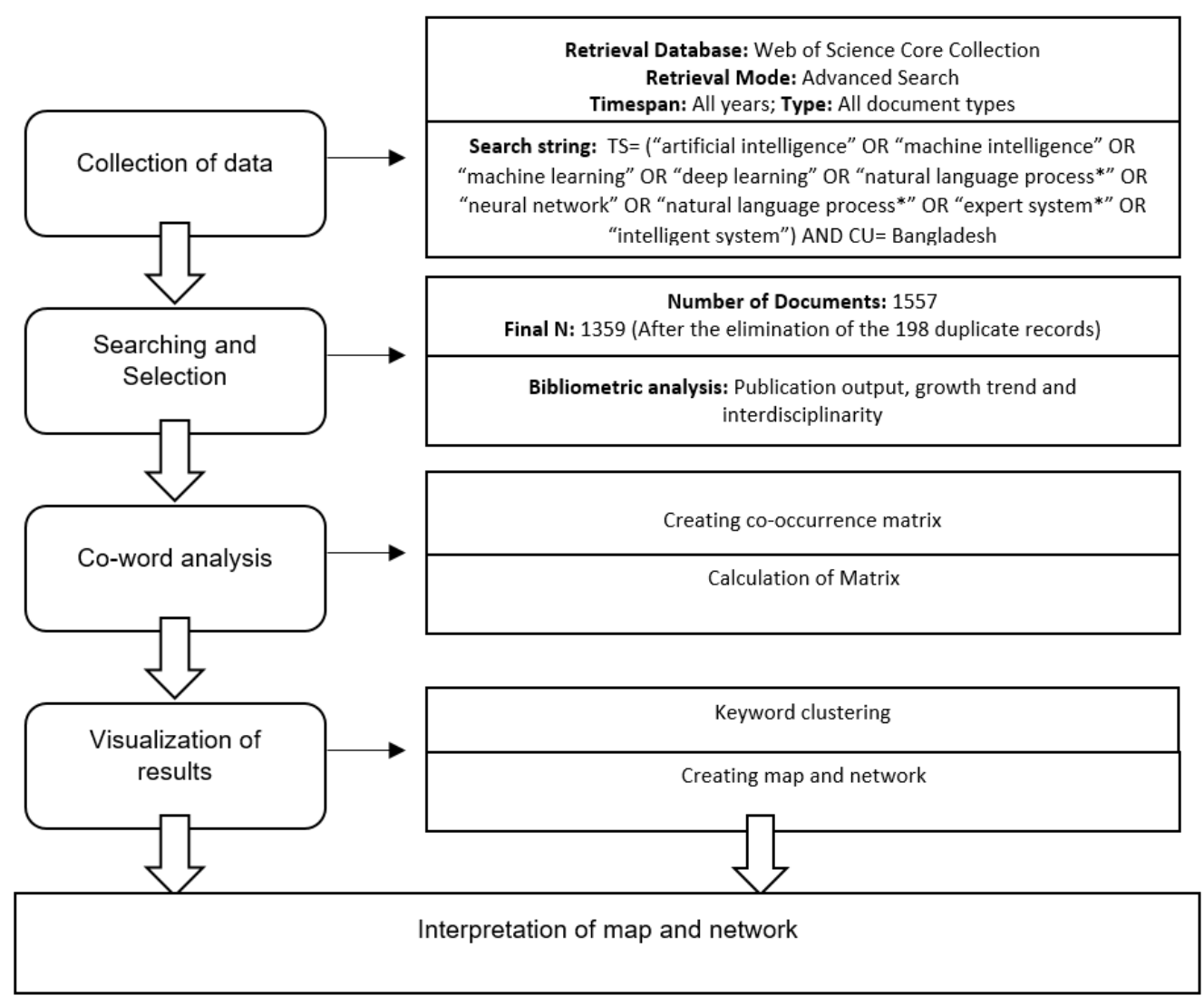

Figure 1: Steps of research methods in this study 
Das \& Islam: Mapping knowledge structure of artificial intelligence research

\section{Results}

\subsection{Literature growth and trend of publication}

As reflected in the WoS, a total of 1,359 research publications were published during 19932020 in AI research domain in Bangladesh. Table 1 shows the distribution of these publications over the studied period, along with their citations (C), and citations per publication (CPP). It is observed that the highest number of papers; 297, was published in 2019 while no single paper published in 1995, 1996 and 1999.

Table 1: Year-wise growth trend of articles

\begin{tabular}{|c|c|c|c|}
\hline $\mathrm{Year}(\mathrm{Y})$ & Publication(P) & Citation(C) & Citation per Publication (CPP) \\
\hline 1993 & 2 & 2 & 1 \\
\hline 1994 & 1 & 0 & 0 \\
\hline 1995 & 0 & 0 & 0 \\
\hline 1996 & 0 & 0 & 0 \\
\hline 1997 & 1 & 0 & 0 \\
\hline 1998 & 2 & 20 & 10 \\
\hline 1999 & 0 & 0 & 0 \\
\hline 2000 & 1 & 130 & 130 \\
\hline 2001 & 3 & 15 & 5 \\
\hline 2002 & 4 & 3 & 0.75 \\
\hline 2003 & 3 & 10 & 3.33 \\
\hline 2004 & 3 & 29 & 9.66 \\
\hline 2005 & 4 & 61 & 15.25 \\
\hline 2006 & 6 & 19 & 3.16 \\
\hline 2007 & 15 & 83 & 5.53 \\
\hline 2008 & 23 & 183 & 7.95 \\
\hline 2009 & 17 & 210 & 12.35 \\
\hline 2010 & 11 & 221 & 20.09 \\
\hline 2011 & 16 & 207 & 12.93 \\
\hline 2012 & 44 & 533 & 12.11 \\
\hline 2013 & 43 & 499 & 11.60 \\
\hline 2014 & 61 & 473 & 7.75 \\
\hline 2015 & 60 & 274 & 4.56 \\
\hline 2016 & 122 & 927 & 7.59 \\
\hline 2017 & 165 & 864 & 5.23 \\
\hline 2018 & 223 & 990 & 4.43 \\
\hline 2019 & 297 & 852 & 2.86 \\
\hline 2020 & 232 & 841 & 3.62 \\
\hline Total & 1359 & 7446 & 296.75 \\
\hline
\end{tabular}


The 1,359 publications received a total of 7,446 citations. 2019 was the most prolific period in terms of citation reaching to 990 , while the year 2000 was most dominant in terms of citations per publication reaching to 130 . However, with the increase of quantity, the number of citations for the period 2007 to 2020 goes upward and it is following the upward trend.

Figure 2 shows that conference-proceedings paper (900) greatly dominate the Bangladeshi presence in the WoS for artificial intelligence research, followed by research article (438), review(17), letter(2), meeting abstract(1) and correction(1). The most cited types of publication in this database are reviews, with a value of 17 for citations per publications, followed by articles (12.97), proceedings (1.64) and letter (1); letters and meeting abstracts are seldom cited.

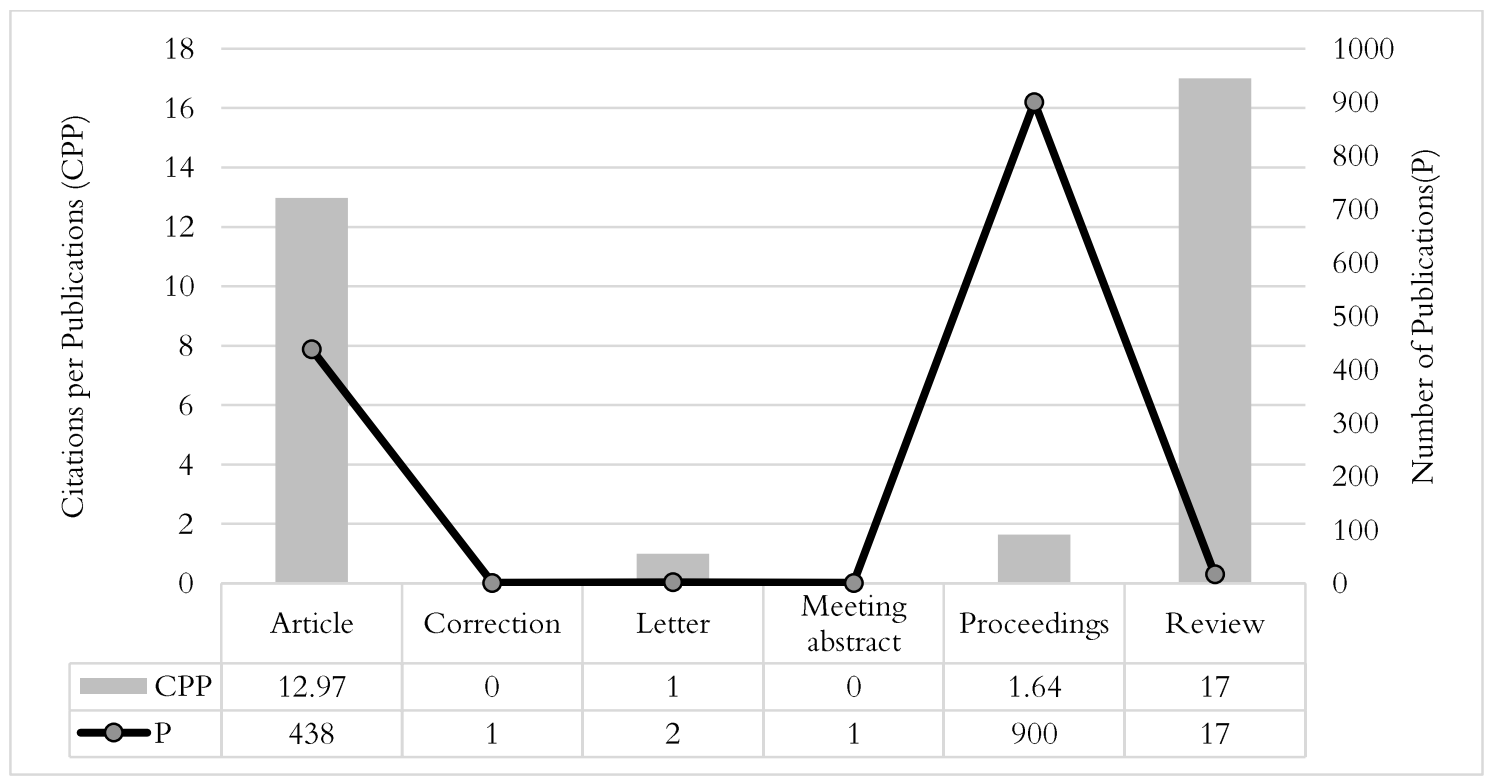

Figure 2: Publication and citation trend in each type of publication

\subsection{Interdisciplinary nature of AI research}

Figure 3 shows the top 10 categories that published at least 30 articles in AI domain from 1993-2020 in Bangladesh. The top categories with 660 publications were electrical \& electronic engineering constitute the highest percentage of $32 \%$. The second highest category was computer science: information systems with 358 publications (15\% of total), followed by computer science: artificial intelligence (16\%), computer science: theory and methods (16\%), and computer science: interdisciplinary applications (5\%), computer science: hardware and architecture (5\%), computer science: software engineering' (3\%), imaging science and photographic technology, engineering, biomedical engineering, multidisciplinary (3\%) each. 


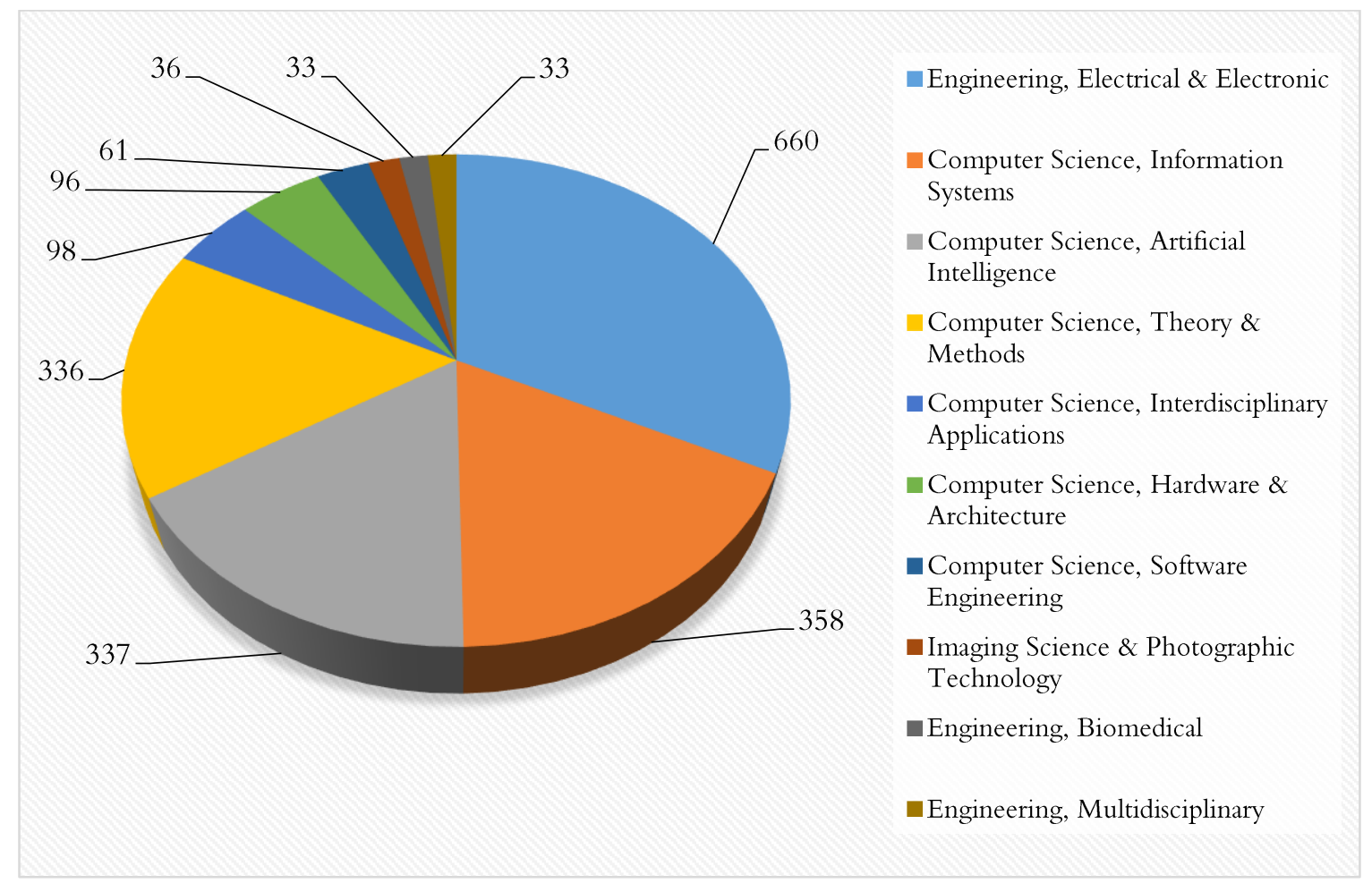

Figure 3: Top $10 \mathrm{Web}$ of Science subject category

\subsection{Research hotspots in artificial intelligence research}

To show the research hotspots in artificial intelligence domain, keyword co-occurrence analysis and keyword cluster analysis have been conducted using VOSviewer. A total of 152 keywords with their co-occurrence threshold $\geq 5$ were identified and brought into the visualization of this study (Table 2). These keywords were primarily classified into 12 clusters through the calculation of co-occurrence matrix. These co-words with higher frequency are often the hot topics in a field. Different colors represent different clusters or sets. Additionally, the size of a node indicates its frequency of being cited. Moreover, the distance between each node one from another indicates the extent of strength of their relationship (shorter distance indicated close relationship, whereas a longer distance refers to remote).

From Table 2, Cluster 1 has the most prevalent number of keywords, indicating that this cluster is the most consolidated research area that pays more attention from the AI researcher in Bangladesh. Cluster 1 contains 26 keywords, for example: artificial intelligence, Bangladesh, cellular automata, climate-change, face recognition, fuzzy inference system, fuzzy logic, genetic algorithm, Geographic Information System (GIS), index, K-nearest neighbor, logistic-regression, model, modeling, neural network, optimization, prediction, prediction model, regression, remote sensing, renewable energy, simulation, surfaceroughness, steel, uncertainty, validation etc. So, it is evident that research topics from the $1^{\text {st }}$ 
cluster have paid close attention to AI research in Bangladesh. Cluster 2 includes the research on Bangla, deep belief network, deep neural network, fake news, hidden Markov model, human computer interaction, image processing, image recognition, information extraction, machine learning, machine translation, naive Bayes, natural language processing, opinion mining, optical character recognition, pattern recognition, sentiment analysis, social media, speech recognition, stylometry, supervised learning, support vector machine, text classification etc. Cluster 3 contains 19 research topics i.e. artificial neural network, classification, computational intelligence, cyber security, data mining, decision trees, diabetes prediction, feature extraction, forecasting, healthcare, IoT, load forecasting, learning, machine learning algorithm, predictive models, signal processing, smart grid, statistics, time-series analysis and so on. Cluster 4 includes 17 research topics including character recognition, character segmentation, classifier, cloud computing, convolutional neural network, data augmentation, deep learning, image classification, information technology, object detection, principal component analysis, raspberry pi, recurrent neural network, reinforcement learning, system, task analysis, transfer learning etc. Cluster 5 and 6 both contains 14 research topics i.e. algorithms, back propagation, biometrics, braincomputer interface, electroencephalography, emotion recognition, empirical model decomposite, entropy, generalization ability, gesture recognition, image segmentation, sign language, training, wavelet transform, attributes, children, classification algorithm, correlation, diagnosis, discrete wavelet transform, disease, epilepsy, feature election, framework, identification, missing values, risk, sequence so on. Rest of the clusters also contain significant research keywords (See Table 2).

Table 3 shows the frequency distribution of top 10 co-words. Based on the VOSviewer manual, apiece link has its own strength, characterized by a positive arithmetical value. The upper the value, the robust the link is. The total link strength signposts the number of publications where two keywords follow each other with distinct connection. In this study research hotspot mainly concentrated on the artificial intelligence, machine learning, classification, neural network, deep learning, artificial neural network, convolutional neural network, support vector machine, prediction and data mining. Artificial intelligence has the highest link, occurrences and link strength with 171172 and 548 respectively. It proves that it is the most centralized and interconnected co-word over the studied period in AI domain in Bangladesh.

Figure 4 displays a co-word map with the most widespread keywords in the publications for the studied period. It shows the frequency of keywords along with their co-occurrences and intensity through colors depicting it as a heat map. Intense color suggests the frequency of keywords. In the center of each intense color, there is a keyword which is labeled in larger font size. It signifies that these groups of keywords performed more commonly and co-occurred with a greater number of other keywords in the texts or literatures. Keywords that are generally co-occurred, more frequently are situated to be nearer in the map. 
Das \& Islam: Mapping knowledge structure of artificial intelligence research

Table 2: 12 cluster of artificial intelligence research in Bangladesh on VOSviewer Matrix

\begin{tabular}{|c|c|c|}
\hline $\begin{array}{l}\text { Cluster } \\
\text { Number }\end{array}$ & $\begin{array}{l}\text { Number } \\
\text { of } \\
\text { Keywords }\end{array}$ & Keywords in the Cluster \\
\hline 1 & 26 & $\begin{array}{l}\text { artificial intelligence, Bangladesh, cellular automata, climate-change, } \\
\text { face recognition, fuzzy inference system, fuzzy logic, genetic algorithm, } \\
\text { GIS, index, K-nearest neighbor, logistic-regression, model, modeling, } \\
\text { neural network, optimization, prediction, prediction model, regression, } \\
\text { remote sensing, renewable energy, simulation, surface-roughness, steel, } \\
\text { uncertainty, validation }\end{array}$ \\
\hline 2 & 23 & $\begin{array}{l}\text { Bangla, deep belief network, deep neural network, fake news, hidden } \\
\text { markov model, human computer interaction, image processing, image } \\
\text { recognition, information extraction, machine learning, machine } \\
\text { translation, naive bayes, natural language processing, opinion mining, } \\
\text { optical character recognition, pattern recognition, sentiment analysis, } \\
\text { social media, speech recognition, stylometry, supervised learning, } \\
\text { support vector machine, text classification }\end{array}$ \\
\hline 3 & 19 & $\begin{array}{l}\text { artificial neural network, classification, computational intelligence, } \\
\text { cyber security, data mining, decision trees, diabetes prediction, feature } \\
\text { extraction, forecasting, healthcare, IoT, load forecasting, learning, } \\
\text { machine learning algorithm, predictive models, signal processing, smart } \\
\text { grid, statistics, time-series analysis }\end{array}$ \\
\hline 4 & 17 & $\begin{array}{l}\text { character recognition, character segmentation, classifier, cloud } \\
\text { computing, convolutional neural network, data augmentation, deep } \\
\text { learning, image classification, information technology, object detection, } \\
\text { principal component analysis, raspberry pi, recurrent neural network, } \\
\text { reinforcement learning, system, task analysis, transfer learning }\end{array}$ \\
\hline 5 & 14 & $\begin{array}{l}\text { algorithms, backpropagation, biometrics, brain-computer interface, } \\
\text { electroencephalography, emotion recognition, empirical model } \\
\text { decomposite, entropy, generalization ability, gesture recognition, image } \\
\text { segmentation, sign language, training, wavelet transform }\end{array}$ \\
\hline 6 & 14 & $\begin{array}{l}\text { attributes, children, classification algorithm, correlation, diagnosis, } \\
\text { discrete wavelet transform, disease, epilepsy, feature election, } \\
\text { framework, identification, missing values, risk, sequence }\end{array}$ \\
\hline 7 & 11 & $\begin{array}{l}\text { accuracy, adaboost, bagging, boosting, classifiers, design, diversity, } \\
\text { ensemble, ensemble classifier, fusion, random forest }\end{array}$ \\
\hline 8 & 9 & $\begin{array}{l}\text { clustering, context-aware computing, data science, discovery, } \\
\text { intelligent systems, personalization, predictive analytics, smartphone, } \\
\text { user behavior modelling }\end{array}$ \\
\hline 9 & 8 & $\begin{array}{l}\text { coronavirus, covid- } 19 \text {, ensemble learning, imbalanced data, k-means } \\
\text { clustering, linear regression, sars-cov- } 2 \text {, smote }\end{array}$ \\
\hline 10 & 6 & $\begin{array}{l}\text { breast cancer, computer-aided diagnosis, confusion matrix, cross } \\
\text { validation, MRI, traffic sign recognition }\end{array}$ \\
\hline 11 & 3 & collaborative filtering, matrix factorization, recommender system \\
\hline 12 & 2 & affective computing, HCI \\
\hline
\end{tabular}


Table 3: Frequency distribution of top ten co-words

\begin{tabular}{clcccc}
\hline Rank & Keyword & $\begin{array}{c}\text { Cluster } \\
\text { Number }\end{array}$ & Links & Occurrences & $\begin{array}{c}\text { Total link } \\
\text { strength }\end{array}$ \\
\hline 1 & artificial intelligence & 1 & 171 & 172 & 548 \\
2 & machine learning & 2 & 137 & 160 & 343 \\
3 & classification & 3 & 160 & 152 & 538 \\
4 & neural network & 1 & 76 & 145 & 357 \\
5 & deep learning & 4 & 128 & 134 & 358 \\
6 & artificial neural network & 3 & 95 & 132 & 279 \\
7 & convolutional neural network & 4 & 73 & 78 & 261 \\
8 & support vector machine & 2 & 59 & 69 & 145 \\
9 & prediction & 1 & 109 & 59 & 185 \\
10 & data mining & 3 & 62 & 52 & 120 \\
\hline
\end{tabular}

As the size of nodes marks the frequency of keywords, the keyword with higher frequency produces greater size of node. The width of line is proportionate to the vicinity between two keywords. The thicker line between two words denotes the close association. As shown in Figure 4, artificial intelligence node has the prime size demonstrating that it has the highest frequency of keywords. Machine learning and classification are the second and the third main keywords (the largest circle of each color). Neural network, deep learning, artificial neural network, convolutional neural network, support vector machine, prediction and data mining also have higher frequency. The thicker lines between two keywords, such as artificial intelligence and machine learning reveals closer relationship. On the contrary, a community of keywords, for example-discovery, personalization, data science, predictive analytics, smartphone, user behavior modeling that have lower frequency as its size of node is small. It proves that these topics are partially disconnected from the rest of the graph. And there are some strokes that are not connected with other keywords. It includes the list asbehavior, context-aware computing etc. indicating these keywords are in the margin bearing no relatedness within the research field.

To plot the area or spot of highly frequent keywords, density visualization of map has been drawn in this study. Individual point in the map has a color that is actually determined by the thickness of items at that particular point. This color generally ranges from red to blue. The larger the number of items in the quarter of a point and the higher the weightiness of the items of that particular quarter, the nearer the color of the point is to red. Similarly, the lesser the number of items in the region of a point and the lower the weights of that regional items, the nearer the color of the point is to the blue. From the density visualization (Figure 5), it is found that Artificial Intelligence, Machine Learning, Data Mining, and Transfer Learning have a high density demonstrating these keywords have a robust relationship with each other. So, these are the more mature and well-developed study on the theme, as it 
carries the higher density. On the other hand, keywords in red area and green area in far location depicts that there are some research fields tend to be nascent.
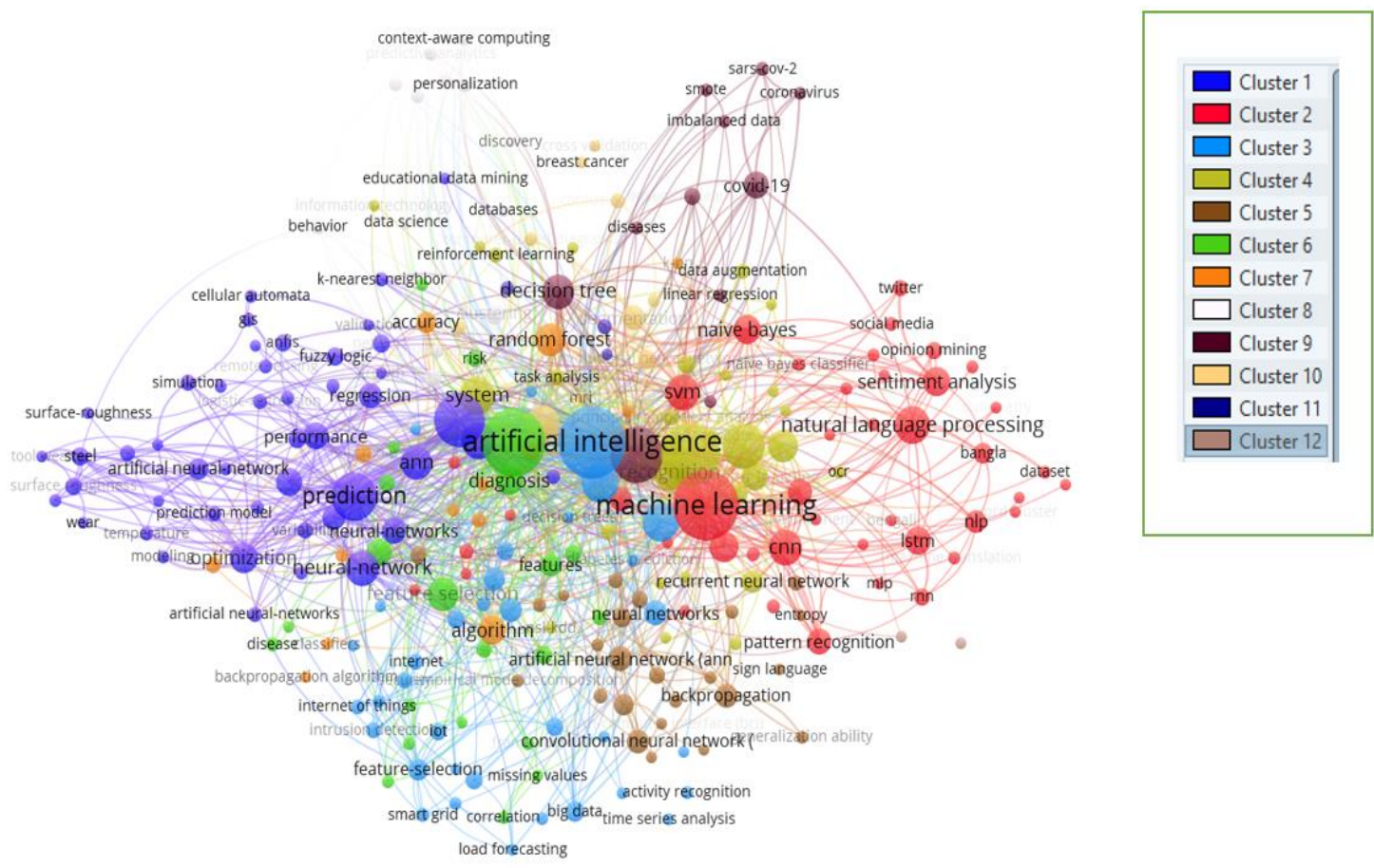

Figure 4: The cluster map of keyword co word analysis based on document-weights (Please view the online version for a true visualization of the figure)

\section{Discussion}

The aim of this study was to visualize the knowledge structure of artificial intelligence (AI) field in Bangladesh during the period of 1993-2020 using bibliometrics through co-word analysis. Summary of the findings are:

- AI researchers of Bangladesh produced highest number of publications in the year of 2019, while the highest citation has been achieved in the year 2018.

- Conference paper has ranked first with 900 publications among other publication types, while research article has ranked second with 438 publications.

- The top WoS category of subject is Electrical \& Electronic Engineering that means researchers from this filed have produced the highest percentage with 660 publications. On the other hand, the category 'Computer science: software engineering', 'Imaging science \& photographic technology', 'Biomedical 
engineering' and 'Multidisciplinary' each has produced the lowest number of publication (3\%).

- The number of themes spotted during the studied period shows a surge in the number of papers published by the AI researcher in Bangladesh. Using the VOSviewer software, this study has uncovered an understanding on the development of AI domain in Bangladesh over the years. According to cluster analysis for 152 keywords shows that the AI research domain of Bangladesh are diverse in nature. About 12 sub-domain clusters have been identified under the artificial intelligence knowledge domain, which epitomize a great research direction.

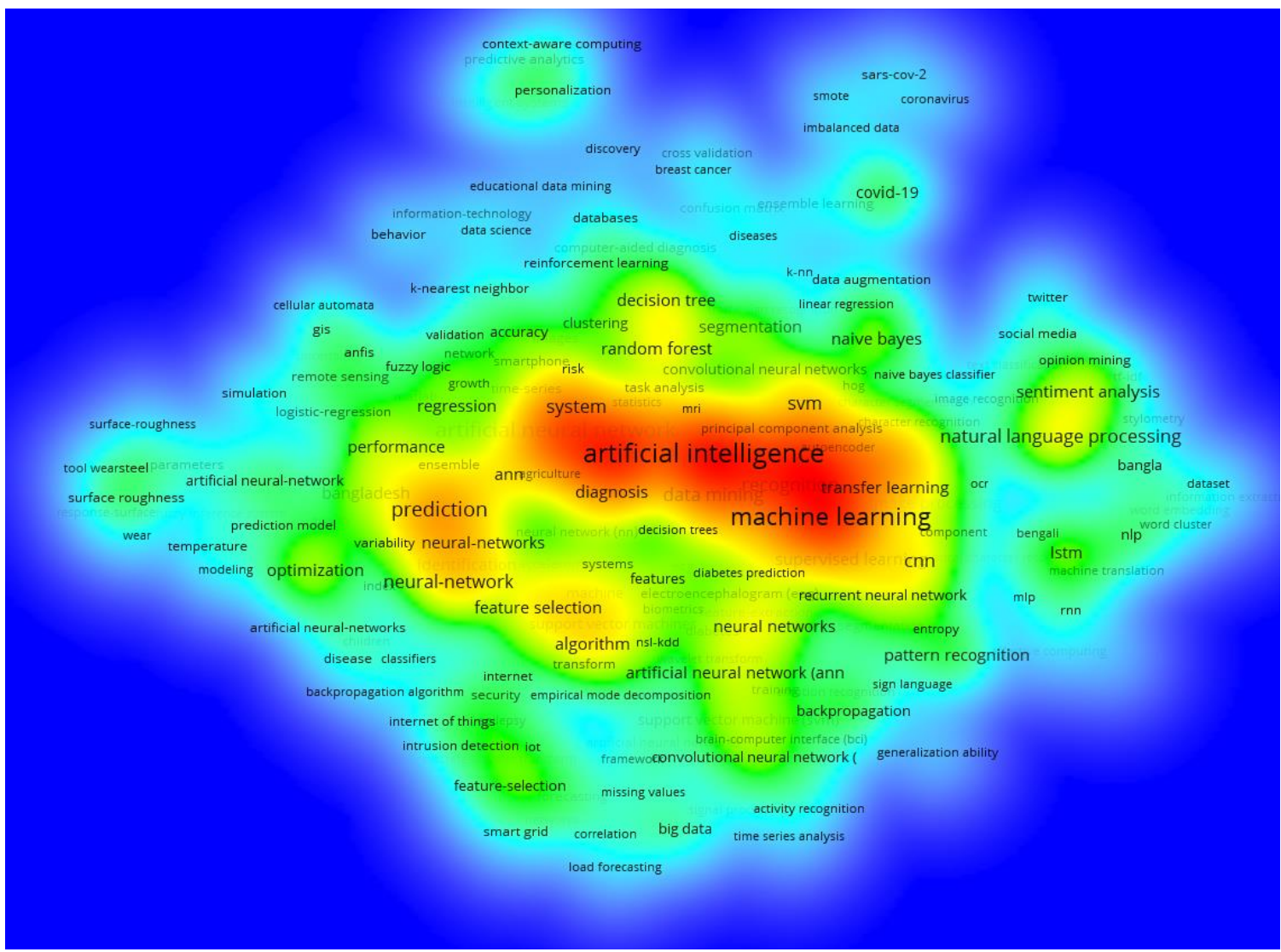

Figure 5: Density visualization map of keyword co word analysis based on documentweights (Please view the online version for a true visualization of the figure)

- From the findings, it is evident that, 'artificial intelligence', 'machine learning', 'classification', 'neural network', 'deep learning', 'artificial neural network', 'convolutional neural network', 'support vector machine', 'prediction' and 'data mining' are the hot topics during the period of studied time. However, the evolution 
Das \& Islam: Mapping knowledge structure of artificial intelligence research

trends in the density visualization suggest that many research fields within AI in Bangladesh are still immature.

\section{Limitation and implications of the study}

This study is not without limitations. First, we retrieved the literature only from WoS. Second, we used VOSviewer software tools for analyzing the whole dataset. Generally, VOSviewer aims to avoid overlapping terms. If multiple terms are positioned adjacent to each other, only one of them is shown, others are not. In that way, the software prevents terms from overlapping each other. When the user zooms in, more space becomes available in the visualization, and therefore more terms will become visible. So, the overlapping terms remain invisible sometimes. Moreover, this study did not take into consideration the quality of research papers. Notwithstanding its limitations, this study may have potential usefulness in uncovering the AI research fields' intellectual structure within a discipline. This will help researchers to understand the current state of the art of artificial intelligence for anticipating future innovation pathways of AI field in Bangladesh.

\section{References}

1. Callon, M., Rip, A., \& Law, J. (Eds.). (1986). Mapping the dynamics of science and technology: Sociology of science in the real world [SpringerLink version]. doi: 10.1007/978-1-349-074082.

2. Cambrosio, A., Limoges, C., Courtial, J., \& Laville, F. (1993). Historical scientometrics? Mapping over 70 years of biological safety research with coword analysis. Scientometrics, 27(2), 119-143. doi:10.1007/BF02019280

3. Chen, C. (2006). CiteSpace II: Detecting and visualizing emerging trends and transient patterns in scientific literature. Journal of the American Society for information Science and Technology, 57(3), 359-377. doi:10.1002/asi.20317

4. Chen, C. (2013) Mapping scientific frontiers: The quest for knowledge visualization (2nd ed.). London, England: Springer-Verlag.

5. Chen, C., Dubin, R., \& Schultz, T. (2015). Science mapping. In Mehdi Khosrow-Pour (Ed.), Encyclopedia of Information Science and Technology (3rd ed.), (pp. 4171-4184). IGI Global. doi: 10.4018/978-1-4666-5888-2.ch410

6. Copeland, B. J. (1993). Artificial intelligence: A philosophical introduction. Oxford, UK; Cambridge, Mass: Blackwell.

7. Coulter, N., Monarch, I., \& Konda, S. (1998). Software engineering as seen through its research literature: A study in co-word analysis. Journal of the American Society for Information Science, 49(13), 1206-1223. doi:10.1002/(SICI)1097-4571(1998)49:13<1206::AIDASI7>3.0.CO;2-F

8. Deka, P., \& Sarmah, M. (2020). Co-occurrence of keywords in ecocriticism research publications: An analytical approach. Library Philosophy and Practice, 1-8. Retrieved from https://digitalcommons.unl.edu/libphilprac/4452

9. Ding, Y., Chowdhury, G. G., \& Foo, S. (2001). Bibliometric cartography of information retrieval research by using co-word analysis. Information Processing \& Management, 37(6), 817842. doi:10.1016/S0306-4573(00)00051-0 
10. Dong, W. (2009). Analysis on hotspot of digital library in home during 10 years based on coword analysis. Document Information \& Knowledge, 5, 58-63.

11. Eom, S. B. (Ed.). (2008). Author Cocitation Analysis: Quantitative methods for mapping the intellectual structure of an academic discipline. Hershey, PA: IGI Global. http://doi:10.4018/978-1-59904-738-6

12. Fosso Wamba, S., Bawack, R. E., Guthrie, C., Queiroz, M. M., \& Carillo, K. D. A. (2021). Are we preparing for a good AI society? A bibliometric review and research agenda. Technological Forecasting and Social Change, 164, 120482. doi:10.1016/J.TECHFORE.2020.120482

13. Garfield, E. (1972). Citation analysis as a tool in journal evaluation. Science, 178(4060), 471479. Retrieved from https://www.jstor.org/stable/1735096

14. Hu, C. P., Hu, J. M., Deng, S. L., \& Liu, Y. (2013). A co-word analysis of library and information science in China. Scientometrics, 97(2), 369-382. doi:10.1007/s11192-013-10767

15. Hu, J., \& Zhang, Y. (2015). Research patterns and trends of recommendation system in China using co-word analysis. Information processing \& management, 51(4), 329-339. doi:10.1016/j.ipm.2015.02.002

16. Hummon, N. P., \& Dereian, P. (1989). Connectivity in a citation network: The development of DNA theory. Social networks, 11(1), 39-63. doi:10.1016/0378-8733(89)90017-8

17. Jacobs, D., Raghava, S. S., \& Pichappan, P. (2006). Text mining using lexical nets: An analysis of word relations. Presented at the Fourth International Conference on Computer Science and Information Technology.

18. Kevork, E. K., \& Vrechopoulos, A. P. (2009). CRM literature: Conceptual and functional insights by keyword analysis. Marketing Intelligence \& Planning, 27(1), 48-85. doi:10.1108/02634500910928362

19. Khasseh, A. A., Soheili, F., Moghaddam, H. S., \& Chelak, A. M. (2017). Intellectual structure of knowledge in iMetrics: A co-word analysis. Information processing \& management, 53(3), 705-720. doi:10.1016/j.ipm.2017.02.001

20. Li, B. H., Hou, B. C., Yu, W. T., Lu, X. B., \& Yang, C. W. (2017). Applications of artificial intelligence in intelligent manufacturing: A review. Frontiers of Information Technology \& Electronic Engineering, 18(1), 86-96. doi:10.1631/FITEE.1601885

21. Liu, G. Y., Hu, J. M., \& Wang, H. L. (2012). A co-word analysis of digital library field in China. Scientometrics, 91(1), 203-217. doi:10.1007/s11192-011-0586-4

22. Luger, G. F. (2005). Artificial intelligence: Structures and strategies for complex problem solving (5th ed). Harlow, England; New York: Addison-Wesley.

23. Lungarella, M., Iida, F., Bongard, J. C., \& Pfeifer, R. (2007). AI in the 21st century - with historical reflections. In M. Lungarella, F. Iida, J. Bongard, \& R. Pfeifer (Eds.), 50 Years of Artificial Intelligence (pp. 1-8). Berlin, Heidelberg: Springer. doi:10.1007/978-3-540-77296$5 \_1$

24. McCarthy, J. (2007). What is artificial intelligence?. Retrieved from http://wwwformal.stanford.edu/jmc/whatisai.pdf

25. Nilsson, Nils J. 1998. Artificial intelligence: A new synthesis. San Francisco, CA: Morgan Kaufmann. 
Das \& Islam: Mapping knowledge structure of artificial intelligence research

26. Peters, H. P. F., \& Van Raan, A. F. J. (1991). Structuring scientific activities by co-author analysis: An expercise on a university faculty level. Scientometrics, 20(1), 235-255. doi:10.1007/BF02018157

27. Poole, D. L., Mackworth, A. K., \& Goebel, R. (1998). Computational intelligence: A logical approach. New York: Oxford University Press.

28. Qiu, J., Dong, K., Yang, S., \& Zhao, R. (2017). Informetrics: Theory, methods and applications. Singapore: Springer.

29. Rip, A., \& Courtial, J. (1984). Co-word maps of biotechnology: An example of cognitive scientometrics. Scientometrics, 6(6), 381-400. doi: 10.1007/BF02025827

30. Ritzhaupt, A. D., Stewart, M., Smith, P., \& Barron, A. E. (2010). An investigation of distance education in North American research literature using co-word analysis. International Review of Research in Open and Distributed Learning, 11(1), 37-60. doi:10.19173/irrodl.v11i1.763

31. Rokaya, M., Atlam, E., Fuketa, M., Dorji, T. C., \& Aoe, J. (2008). Ranking of field association terms using co-word analysis. Information Processing \& Management, 44(2), 738-755. doi:10.1016/j.ipm.2007.06.001

32. Ronda-Pupo, G. A., \& Guerras-Martin, L. Á. (2012). Dynamics of the evolution of the strategy concept 1962-2008: A co-word analysis. Strategic Management Journal, 33(2), 162-188. doi:10.1002/smj.948

33. Russell, S., \& Norvig, P. (2002). Artificial intelligence: A modern approach (2 ${ }^{\text {nd }}$ ed.). Upper Saddle River, NJ: Prentice Hall.

34. Su, N. (2009). Analysis of subjects and development in digital libraries research based on cowords analysis method. Journal of Intelligence, 28(6), 15-19.

35. Van Eck, N. J., \& Waltman, L. (2010). Software survey: VOSviewer, a computer program for bibliometric mapping. scientometrics, 84(2), 523-538. doi:10.1007/s11192-009-0146-3

36. Wang, L. Y., Zhang, Z. Q., \& Wei, J. Z. (2011). A study on foreign research subjects of library and information science based on the co-word analysis during the last ten years. Journal of intelligence (in China), 30(3), 50-58.

37. Xiang, C., Wang, Y., \& Liu, H. (2017). A scientometrics review on nonpoint source pollution research. Ecological Engineering, 99, 400-408. doi:10.1016/j.ecoleng.2016.11.028

\section{Author biography}

Rajesh Kumar Das is an Assistant Professor in the Institute of Information Sciences at Noakhali Science and Technology University. Mr. Das did his bachelor and master from University of Dhaka. His primary research interests focus on the linkages among scientific domains, using science mapping and metascience methodologies. He has also concurrent research in information behavior, data science, scholarly communication, and knowledge transfer. He has presented papers at conferences home and abroad, published papers in international peer-reviewed journals, and contributed articles to newspapers, magazines etc. He has served as research editor, chair of conference sessions, and officer of Special Interest Groups. 
IJIKS, Vol. 1 No. 1 (2021)

Mohammad Sharif Ul Islam is an Assistant Professor at Information Science and Library Management Department, University of Dhaka. He spends most of his time teaching and research along with numerous voluntarily and social works. Mr. Islam has achieved his MSC in Data Science from the University of Sheffield, UK under the Commonwealth Scholarship. Prior to that, he received both of his Honors and Masters degree from University of Dhaka. He is a strong voice of equality and justice, and thus, his research interest lies with any contemporary social issues, i.e., health, education, information fits within the spectrum of quantitative research.

\section{Corresponding author}

Rajesh Kumar Das can be contacted at: rajesh@,nstu.edu.bd 